\title{
Searches for magnetic monopoles and beyond with MoEDAL at the LHC
}

\author{
Vasiliki A. Mitsou ${ }^{1, *}$ \\ On behalf of the MoEDAL Collaboration \\ ${ }^{1}$ Instituto de Física Corpuscular (IFIC), CSIC - Universitat de València, \\ Parc Científic de la U.V., C/ Catedrático José Beltrán 2, \\ E-46980 Paterna (Valencia), Spain
}

\begin{abstract}
The MoEDAL experiment at the LHC is optimised to detect highly-ionising particles such as magnetic monopoles, dyons and (multiply) electrically-charged stable massive particles predicted in a number of theoretical scenarios. MoEDAL, deployed in the LHCb cavern, combines passive nuclear track detectors with magnetic monopole trapping volumes, while cavern backgrounds are being monitored with an array of MediPix detectors. The detector concept and its physics reach is presented with emphasis given to recent results on monopole searches providing the best limits on high magnetic charges in colliders. The potential to search for heavy, long-lived supersymmetric electrically-charged particles and multi-charged states is also discussed.
\end{abstract}

\section{Introduction}

MoEDAL (Monopole and Exotics Detector at the LHC) [1, 2], the $7^{\text {th }}$ experiment at the Large Hadron Collider (LHC) [3], is designed to search for manifestations of new physics through highly-ionising particles in a manner complementary to ATLAS, CMS and ALICE [4]. The most important motivation for the MoEDAL experiment is to pursue the quest for magnetic monopoles and dyons at LHC energies. In addition, the experiment is designed to search for any massive, stable or long-lived, slow-moving particle [5] with single or multiple electric charges that arise in various scenarios of physics beyond the Standard Model (SM). For an extended and detailed account of the MoEDAL discovery potential, the reader is referred to the MoEDAL Physics Review [6]. Emphasis is given here on recent MoEDAL results, based on the exposure of magnetic monopole trapping volumes to $8 \mathrm{TeV}$ proton-proton collisions during Run 1 and $13 \mathrm{TeV}$ collisions during Run 2.

The structure of this paper is as follows. In Section 2, magnetic monopoles and monopolia are briefly discussed. Section 3 provides a brief description of the MoEDAL detector, whilst Section 4 presents the MoEDAL results on monopole searches. Section 5 is dedicated to supersymmetric models predicting massive (meta)stable states. The paper concludes with a summary and an outlook in Section 6.

\footnotetext{
*e-mail: vasiliki.mitsou@ific.uv.es
} 


\section{Magnetic monopoles}

The MoEDAL detector is designed to fully exploit the energy-loss mechanisms of magnetically charged particles [7-10] in order to optimise its potential to discover these messengers of new physics. There are various theoretical scenarios in which magnetic charge would be produced at the LHC [6]: (light) 't Hooft-Polyakov monopoles [9, 11], electroweak monopoles [12-14], global monopoles [15-18] and monopolium [8, 19-21]. Magnetic monopoles that carry a non-zero magnetic charge and dyons possessing both magnetic and electric charge are among the most fascinating hypothetical particles. Even though there is no generally acknowledged empirical evidence for their existence, there are strong theoretical reasons to believe that they do exist, and they are predicted by many theories including grand unified theories and superstring theory $[22,23]$.

The theoretical motivation behind the introduction of magnetic monopoles is the symmetrisation of the Maxwell equations and the explanation of the charge quantisation [7]. Dirac showed that the mere existence of a monopole in the universe could offer an explanation of the discrete nature of the electric charge, leading to the Dirac Quantisation Condition (DQC),

$$
\alpha g=\frac{N}{2} e, \quad N=1,2, \ldots
$$

where $e$ is the electron charge, $\alpha=\frac{e^{2}}{4 \pi \hbar c \varepsilon_{0}}=\frac{1}{137}$ is the fine structure constant (at zero energy, as appropriate to the fact that the DQC pertains to long (infrared) distances from the centre of the monopole), $\varepsilon_{0}$ is the vacuum permittivity, and $g$ is the monopole magnetic charge. In Dirac's formulation, magnetic monopoles are assumed to exist as point-like particles and quantum mechanical consistency conditions lead to Eq. (1), establishing the value of their magnetic charge. Although monopoles symmetrise Maxwell equations in form, there is a numerical asymmetry arising from the DQC, namely that the basic magnetic charge is much larger than the smallest electric charge. A magnetic monopole with a single Dirac charge $\left(g_{\mathrm{D}}\right)$ has an equivalent electric charge of $\beta(137 e / 2)$. Thus for a relativistic monopole the energy loss is around 4,700 times $\left(68.5^{2}\right)$ that of a minimum-ionising electrically-charged particle. The monopole mass remains a free parameter of the theory.

A possible explanation for the lack of experimental confirmation of monopoles is Dirac's proposal $[7,8,19]$ that monopoles are not seen freely because they form a bound state called monopolium [20, 21, 24] being confined by strong magnetic forces. Monopolium is a neutral state, hence it is difficult to detect directly at a collider detector, although its decay into two photons would give a rather clear signal for the ATLAS and CMS detectors [25, 26], which however would not be visible in the MoEDAL detector. Nevertheless according to a novel proposal [27], the LHC radiation detector systems can be used to turn the LHC itself into a new physics search machine by detecting final-state protons $p p \rightarrow p X p(X$ are centrally produced particles), exiting the LHC beam vacuum chamber at locations determined by their fractional momentum losses. At high central masses $M_{X}>150 \mathrm{GeV}$, the photon-photon process dominates making this technique appealing for detecting monopolia, which are expected to be produced in $\gamma \gamma$ fusion [24, 25].

\section{The MoEDAL detector}

The MoEDAL detector [2] is deployed around the intersection region at Point 8 (IP8) of the LHC (IP8) in the LHCb experiment Vertex Locator (VELO) [28] cavern. A three-dimensional depiction of the MoEDAL experiment is presented in Fig. 1. It is a unique and largely passive 
LHC detector comprised of four sub-detector systems. It is worth emphasising that the detector components are renewed and installed/re-installed into upgraded configurations during technical stops throughout Run 1 (2010-2012) and Run 2 (2015-2018).

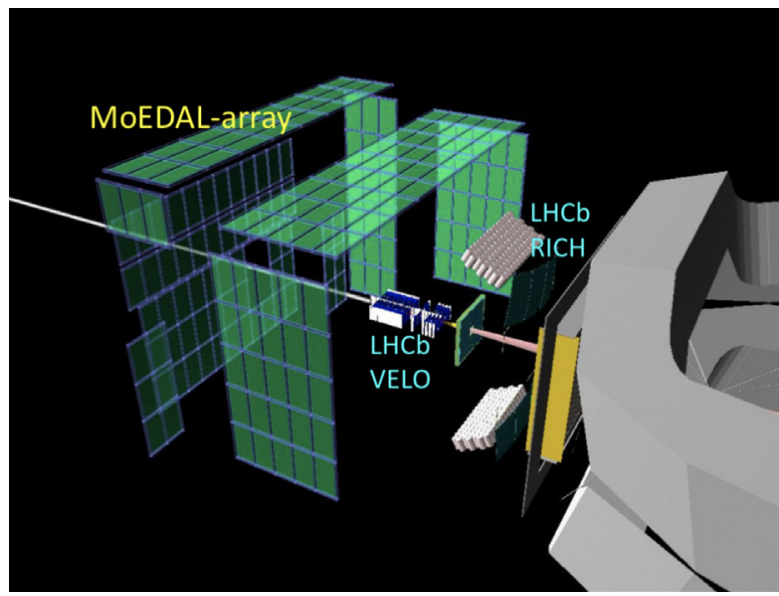

Figure 1. A three-dimensional schematic view of the MoEDAL detector around the LHCb VELO region at Point 8 of the LHC.

\subsection{Low-threshold nuclear track detectors}

The main sub-detector system is made of a large array of CR39®, Makrofol $($ and Lexan $\AA$ plastic stacks surrounding the intersection area. The passage of a highly-ionising particle through these nuclear track detectors (NTDs) is marked by an invisible damage zone along the trajectory. The damage zone is revealed as a cone-shaped etch-pit when the plastic detector is chemically etched. Then the sheets of plastics are scanned looking for aligned etch pits in multiple sheets. The MoEDAL NTDs have a threshold of $Z / \beta \sim 5$, where $Z$ is the charge and $\beta=v / c$ the velocity of the incident particle. During proton-proton collision running, the only source of known particles that are highly ionising enough to leave a track in MoEDAL NTDs are spallation products with range that is typically much less than the thickness of one sheet of the NTD stack. In that case the ionising signature will be that of a very low-energy electrically-charged stopped particle. This signature is distinct to that of a penetrating electrically or magnetically charged particle that will usually traverse every sheet in a MoEDAL NTD stack, accurately demarcating a track that points back to the collision point with a resolution of $\sim 1 \mathrm{~cm}$. The part of the Run 2 NTD deployment which rests on top of the LHCb VELO is visible in Fig. 2. This is the closest possible location to the interaction point and represents a novelty of this run with respect to earlier installations during Run 1.

The basic detector unit of the MoEDAL experiment is a stack of ten sheets of plastic NTDs, consisting of four sheets of CR39®, each $\sim 0.5 \mathrm{~mm}$ thick, three sheets of Makrofol $\AA$ (each $\sim 0.5 \mathrm{~mm}$ thick) with Lexan $囚$ (each $\sim 0.2 \mathrm{~mm}$ thick) forming the first, middle and end sheets of the stack.

\subsection{Very high-charge catcher}

Another new feature of the Run 2 deployment is the installation of a high-threshold NTD array $(Z / \beta \sim 50)$ : the Very High Charge Catcher (VHCC). The VHCC sub-detector, consisting of two flexible low-mass stacks of Makrofol $@$ in an aluminium foil envelope, is deployed in the forward acceptance of the LHCb experiment between the LHCb RICH1 detector and the 


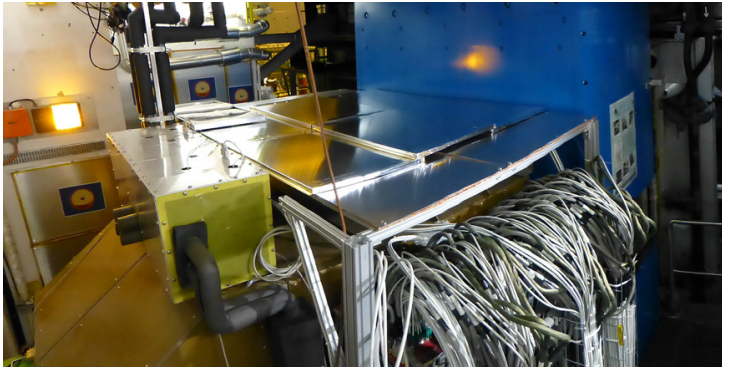

Figure 2. Part of the Run 2 NTD deployment on top of the LHCb VELO.

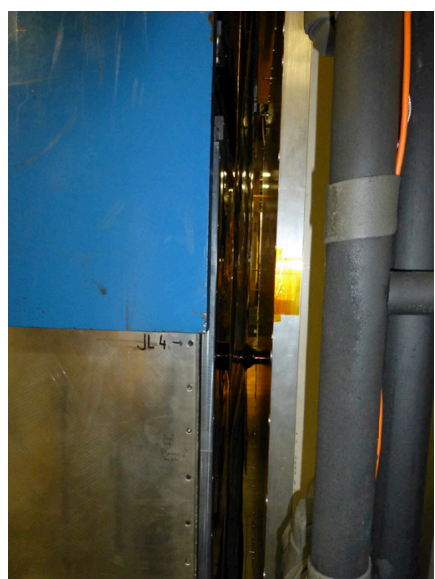

Figure 3. The VHCC between RICH1 and TT installed for Run 2.

Trigger Tracker (TT), as shown in Fig. 3. It is the only NTD (partly) covering the forward region, adding only $\sim 0.5 \%$ to the LHCb material budget while enhancing considerably the overall geometrical coverage of MoEDAL NTDs.

\subsection{Magnetic trappers}

A unique feature of the MoEDAL detector is the use of paramagnetic magnetic monopole trappers (MMTs) to capture electrically- and magnetically-charged highly-ionising particles. Such volumes installed in IP8 for the 2015 proton-proton collisions is shown in Fig. 4. The aluminium absorbers of the MMTs are subject to an analysis looking for magneticallycharged particles at a remote SQUID magnetometer facility [29, 30]. The search for the decays of long-lived electrically charged particles that are stopped in the trapping detectors will subsequently be carried out at a remote underground facility.

A trapping detector prototype was exposed to $8 \mathrm{TeV}$ proton-proton collisions for an integrated luminosity of $0.75 \mathrm{fb}^{-1}$ in 2012. For the 2015 run at $13 \mathrm{TeV}$, the MMT was upgraded to an array consisting of 672 square aluminium rods with dimension $19 \times 2.5 \times 2.5 \mathrm{~cm}^{3}$ for a total mass of $222 \mathrm{~kg}$ in 14 stacked boxes that were placed $1.62 \mathrm{~m}$ from the IP8 LHC interaction point under the beam pipe on the side opposite to the $\mathrm{LHCb}$ detector. The results for the Run 2 configuration are presented in Section 4.

\subsection{TimePix radiation monitors}

The only non-passive MoEDAL sub-detector system comprises an array of TimePix pixel device arrays $(256 \times 256$ square pixels with a pitch of $55 \mu \mathrm{m})$ distributed throughout the MoEDAL cavern at IP8, forming a real-time radiation monitoring system of highly-ionising beam-related backgrounds. A photo of its readout setup for the 2015 installations is shown in Fig. 5. Each pixel of the TimePix chip comprises a preamplifier, a discriminator with threshold adjustment, synchronisation logic and a 14-bit counter. The operation of TimePix in time-over-threshold mode allows a 3D mapping of the charge spreading effect in the whole volume of the silicon sensor, thus differentiating between different types of particles species from mixed radiation fields and measuring their energy deposition [31]. 


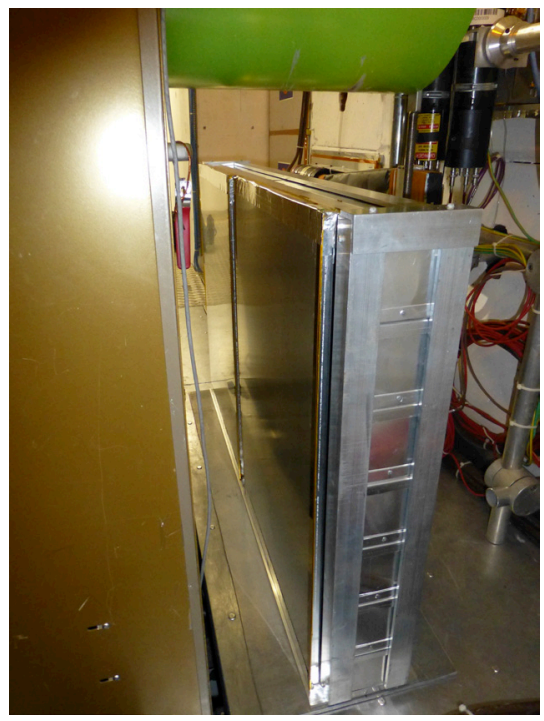

Figure 4. Deployment of the MMT for the LHC Run 2.

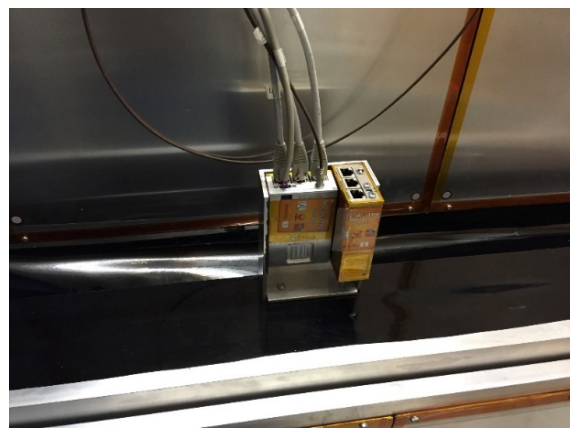

Figure 5. Run 2 deployment of TimePix chips in MoEDAL.

\section{Searches for monopoles in MoEDAL}

The high ionisation of slow-moving magnetic monopoles and dyons, implies quite characteristic trajectories when such particles interact with the MoEDAL NTDs, which can be revealed during the etching process $[2,6]$. In addition, the high magnetic charge of a monopole (which is expected to be at least one Dirac charge $g_{D}=68.5 e$ ( $c f$. Eq. (1)) implies a strong magnetic dipole moment, which in turn may result in a strong binding of the monopole with the ${ }_{13}^{27} \mathrm{Al}$ nuclei of the aluminium MoEDAL MMTs. In such a case, the presence of a monopole trapped in an aluminium bar of an MMT would be detected through the existence of a persistent current, defined as the difference between the currents in the SQUID of a magnetometer before and after the passage of the bar through the sensing coil.

In the context of the MMT exposure during Run 2, no magnetic charge exceeding $0.5 g_{\mathrm{D}}$ was detected in any of the exposed samples when passed through the ETH Zurich SQUID facility, allowing limits to be placed on monopole production. Model-independent crosssection limits have been obtained in fiducial regions of monopole energy and direction for $1 g_{\mathrm{D}} \leq|g| \leq 6 g_{\mathrm{D}}$ with the $8 \mathrm{TeV}$ analysis [32]. Model-dependent cross-section limits are obtained for Drell-Yan (DY) pair production of spin- $1 / 2$ and spin-0 monopoles for $1 g_{\mathrm{D}} \leq|g| \leq 5 g_{\mathrm{D}}$ at $13 \mathrm{TeV}$ [33], as shown in Fig. 6. Caution, however, should be exerted here in the sense that the non-perturbative nature of the large magnetic Dirac charge of the monopole invalidate any perturbative treatment based on Drell-Yan calculations of the pertinent cross sections and hence any result based on the latter is only indicative, due to the lack of any other concrete theoretical treatment. This situation may be resolved if thermal production in heavy-ion collisions - that does not rely on perturbation theory - is considered [34].

The weaker limits for $|g|=g_{\mathrm{D}}$ displayed in Fig. 6 when compared to higher charges are mostly due to loss of acceptance from monopoles punching through the trapping volume. For higher charges, monopoles ranging out before reaching the trapping volume decrease the acceptance for DY monopoles with increasing charge and reaches below $0.1 \%$ for a charge of $6 g_{\mathrm{D}}$. The spin dependence is solely due to the different event kinematics: more central and more energetic monopoles for spin 0 . 

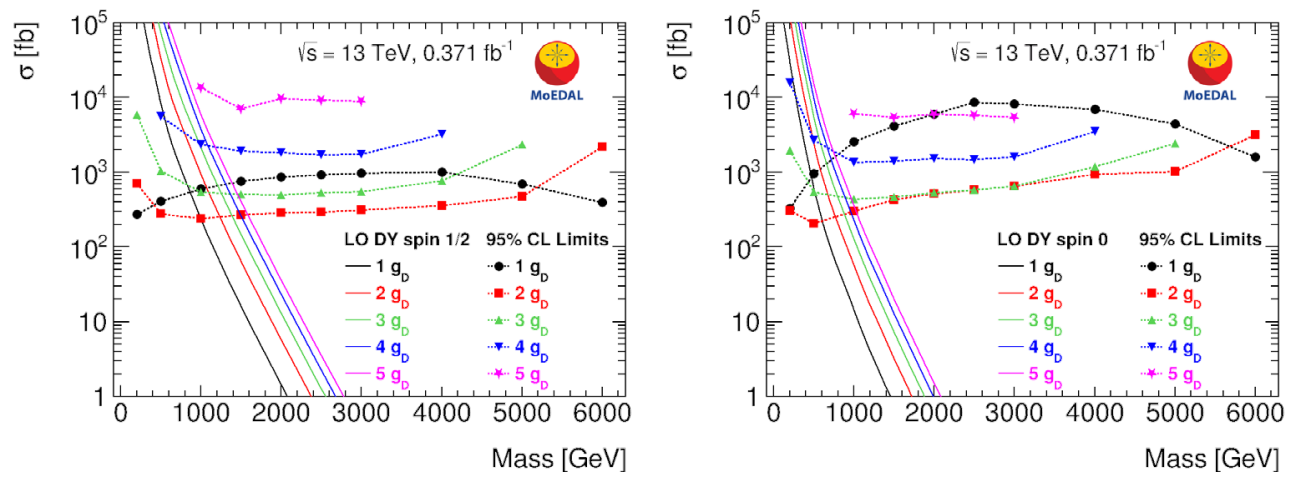

Figure 6. Cross-section upper limits at 95\% confidence level (dotted lines) for DY monopole production as a function of mass for spin- $1 / 2$ (left) and for spin- 0 monopoles (right). The various marker styles and colours correspond to different monopole charges. The solid lines represent DY cross-section calculations at leading order. From Ref. [33].

Under the assumption of Drell-Yan cross sections, mass limits are derived for $1 g_{\mathrm{D}} \leq|g| \leq$ $4 g_{\mathrm{D}}$ at the LHC, complementing previous results from ATLAS Collaboration [35, 36], which placed limits for monopoles with magnetic charge $|g| \leq 1.5 g_{\mathrm{D}}$ (c.f. Fig. 7). The ATLAS bounds are more stringent that those of MoEDAL for $|g|=1 g_{\mathrm{D}}$ due to the higher luminosity delivered in ATLAS and the loss of acceptance in MoEDAL for small magnetic charges. On the other hand, higher charges are difficult to be probed in ATLAS due to the limitations of the electromagnetic-calorimeter-based level-1 trigger deployed for such searches. A comparison of the limits on monopole production cross sections set by other colliders with those set by MoEDAL is presented in Ref. [23], while general limits including searches in cosmic radiation are reviewed in Ref. [37].

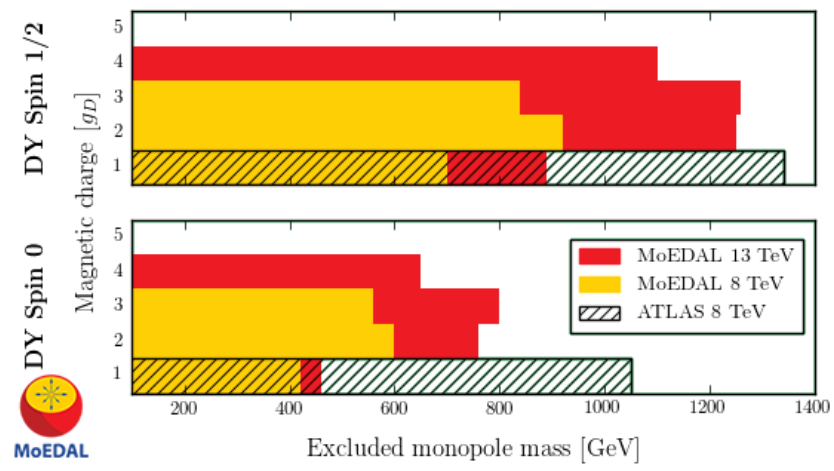

Figure 7. Excluded monopole masses for DY production for spin-1/2 (top) and spin-0 (bottom) monopoles. The MoEDAL results obtained at $8 \mathrm{TeV}$ (yellow, light grey) [32] and $13 \mathrm{TeV}$ (red, dark grey) [33] are superimposed on the ATLAS $8 \mathrm{TeV}$ limits (hatched area) [36]. 


\section{Electrically-charged long-lived particles in supersymmetry}

Supersymmetry (SUSY) is an extension of the Standard Model which assigns to each SM field a superpartner field with a spin differing by a half unit. SUSY provides elegant solutions to several open issues in the SM, such as the hierarchy problem, the identity of dark matter, and the grand unification. SUSY scenarios propose a number of massive slowly moving electrically charged particles. If they are sufficiently long-lived to travel a distance of at least $O(1 \mathrm{~m})$ before decaying and their $Z / \beta \gtrsim 5$, they may be detected in the MoEDAL NTDs. No highly-charged particles are expected in such a theory, but there are several scenarios in which supersymmetry may yield massive, long-lived particles that could have electric charges \pm 1 , potentially detectable in MoEDAL if they are produced with low velocities $(\beta \lesssim 0.2)$.

The lightest supersymmetric particle (LSP) is stable in models where $R$ parity is conserved, where $R$ is a multiplicative quantum number defined as: $R=(-1)^{3 B+L+2 s}$, with $s$ the spin, $B$ the baryon number, and $L$ the lepton number. The LSP should have no strong or electromagnetic interactions, otherwise it would bind to conventional matter and be detectable in anomalous heavy nuclei [38]. Possible weakly-interacting neutral candidates in the Minimal Supersymmetric Standard Model (MSSM) include the sneutrino, which has been excluded by LEP and direct searches, the lightest neutralino $\tilde{\chi}_{1}^{0}$ (a mixture of spartners of the $Z, H$ and $\gamma)$ and the gravitino $\tilde{G}$.

\subsection{Supersymmetric scenarios with $R$-parity violation}

Several scenarios featuring metastable charged sparticles might be detectable in MoEDAL. One such scenario is that $R$ parity may not be exact, since there is no exact local symmetry associated with either $L$ or $B$, and hence no fundamental reason why they should be conserved. One could consider various ways in which $L$ and/or $B$ could be violated in such a way that $R$ is violated, as represented by the following superpotential terms:

$$
W_{R V}=\lambda_{i j k}^{\prime \prime} \bar{U}_{i} \bar{D}_{j} \bar{D}_{k}+\lambda_{i j k}^{\prime} L_{i} Q_{j} \bar{D}_{k}+\lambda_{i j k} L_{i} L_{j} \bar{E}_{k}+\mu_{i} L_{i} H
$$

where $Q_{i}, \bar{U}_{i}, \bar{D}_{i}, L_{i}$ and $\bar{E}_{i}$ denote chiral superfields corresponding to quark doublets, antiquarks, lepton doublets and antileptons, respectively, with $i, j, k$ generation indices. The simultaneous presence of terms of the first and third type in Eq. (2), namely $\lambda$ and $\lambda^{\prime \prime}$, is severely restricted by lower limits on the proton lifetime, but other combinations are less restricted. The trilinear couplings in Eq. (2) generate sparticle decays such as $\tilde{q} \rightarrow \bar{q} \bar{q}$ or $q \ell$, or $\tilde{\ell} \rightarrow \ell \ell$, whereas the bilinear couplings in Eq. (2) generate Higgs-slepton mixing and thereby also $\tilde{q} \rightarrow q \ell$ and $\tilde{\ell} \rightarrow \ell \ell$ decays [39]. For a nominal sparticle mass $\sim 1 \mathrm{TeV}$, the lifetime for such decays would exceed a few nanoseconds for $\lambda, \lambda^{\prime}, \lambda^{\prime \prime}<10^{-8}$.

If $R$ parity is broken, the LSP would be unstable, and might be charged and/or coloured. In the former case, it might be detectable directly at the LHC as a massive slowly-moving charged particle. In the latter case, the LSP would bind with light quarks and/or gluons to make colour-singlet states, the so-called $R$-hadrons, and any charged state could again be detectable as a massive slowly-moving charged particle. If $\lambda \neq 0$, the prospective experimental signature would be similar to a stau next-to-lightest sparticle (NLSP) case to be discussed later. On the other hand, if $\lambda^{\prime}$ or $\lambda^{\prime \prime} \neq 0$, the prospective experimental signature would be similar to a stop NLSP case, yielding the possibility of charge-changing interactions while passing through matter. This could yield a metastable charged particle, created whilst passing through the material surrounding the intersection point, that would be detected by MoEDAL. 


\subsection{Metastable lepton NLSP in the CMSSM with a neutralino LSP}

However, even if $R$ parity is exact, the NLSP may be long lived. This would occur, for example, if the LSP is the gravitino, or if the mass difference between the next-to-lightest supersymmetric particle (NLSP) and the neutralino LSP is small, offering more scenarios for long-lived charged sparticles. In neutralino dark matter scenarios based on the constrained minimal supersymmetric standard model (CMSSM), for instance, the most natural candidate for the NLSP is the lighter stau slepton $\tilde{\tau}_{1}$ [40], which could be long lived if $m_{\tilde{\tau}_{1}}-m_{\tilde{\chi}_{1}^{0}}$ is small. There are several regions of the CMSSM parameter space that are compatible with the constraints imposed by unsuccessful searches for sparticles at the LHC, as well as the discovered Higgs boson mass and the LSP relic density allowed by cosmological observations. It was found in a global analysis that these two possibilities are favoured [41]: (a) a region where the relic density is controlled by rapid annihilation through direct-channel heavy Higgs resonances due to the relatively large higgsino component of the LSP, and (b) a strip where the relic LSP density is reduced by coannihilations with near-degenerate staus and other sleptons.

In the coannihilation region of the CMSSM, the lighter $\tilde{\tau}_{1}$ is expected to be the lightest slepton [40], and the $\tilde{\tau}_{1}-\tilde{\chi}_{1}^{0}$ mass difference may well be smaller than $m_{\tau}$ : indeed, this is required at large LSP masses. In this case, the dominant stau decays for $m_{\tilde{\tau}_{1}}-m_{\tilde{\chi}_{1}^{0}}>$ $160 \mathrm{MeV}$ are expected to be into three particles: $\tilde{\chi}_{1}^{0} v \pi$ or $\tilde{\chi}_{1}^{0} v \rho$. If $m_{\tilde{\tau}_{1}}-m_{\tilde{\chi}_{1}^{0}}<1.2 \mathrm{GeV}$, the $\tilde{\tau}_{1}$ lifetime is calculated to be so long, in excess of $\sim 100 \mathrm{~ns}$, that it is likely to escape the detector before decaying, and hence would be detectable as a massive, slowly-moving charged particle $[42,43]$.

\subsection{Metastable sleptons in gravitino LSP scenarios}

On the other hand, in gravitino dark matter scenarios with more general options for the pattern of supersymmetry breaking, other options appear quite naturally, including the lighter selectron or smuon, or a sneutrino [44], or the lighter stop squark $\tilde{t}_{1}$ [45]. If the gravitino $\tilde{G}$ is the LSP, the decay rate of a slepton NLSP is given by

$$
\Gamma(\tilde{\ell} \rightarrow \tilde{G} \ell)=\frac{1}{48 \pi M_{*}^{2}} \frac{m_{\tilde{\ell}}^{5}}{M_{\tilde{G}}^{2}}\left[1-\frac{M_{\tilde{G}}^{2}}{m_{\tilde{\ell}}^{2}}\right]^{4},
$$

where $M_{*}$ is the Planck scale. Since $M_{*}$ is much larger than the electroweak scale, the NLSP lifetime is naturally very long.

Gravitino (or axino) LSP with a long-lived charged stau may arise in gauge mediation and minimal supergravity models [46]. Large part of the parameter space potentially attractive for long-lived slepton searches with MoEDAL are compatible with cosmological constraints on the dark-matter abundance in superweakly interacting massive particle scenarios [47].

\subsection{Long-lived gluinos in split supersymmetry}

The above discussion has been in the context of the CMSSM and similar scenarios where all the supersymmetric partners of Standard Model particles have masses in the TeV range. Another scenario is "split supersymmetry", in which the supersymmetric partners of quarks and leptons are very heavy, of a scale $m_{s}$, whilst the supersymmetric partners of SM bosons are relatively light [48]. In such a case, the gluino could have a mass in the $\mathrm{TeV}$ range and hence be accessible to the LHC, but would have a very long lifetime:

$$
\tau \approx 8\left(\frac{m_{s}}{10^{9} \mathrm{GeV}}\right)^{4}\left(\frac{1 \mathrm{TeV}}{m_{\tilde{g}}}\right)^{5} \mathrm{~s} .
$$


Long-lived gluinos would form long-lived gluino R-hadrons including gluino-gluon (gluinoball) combinations, gluino- $q \bar{q}$ (mesino) combinations and gluino-qqq (baryino) combinations. The heavier gluino hadrons would be expected to decay into the lightest species, which would be metastable, with a lifetime given by Eq. (4), and it is possible that this metastable gluino hadron could be charged.

In the same way as stop hadrons, gluino hadrons may flip charge through conventional strong interactions as they pass through matter, and it is possible that one may pass through most of a conventional LHC tracking detector undetected in a neutral state before converting into a metastable charged state that could be detected by MoEDAL.

\subsection{MoEDAL complementarity to ATLAS, CMS and ALICE}

There are several considerations supporting the complementary aspects of MoEDAL w.r.t. ATLAS and CMS when discussing the observability of (meta-)stable massive electricallycharged particles. Most of them stem from MoEDAL being "time-agnostic" due to the passive nature of its detectors. Therefore a signal from very slowly moving particles will not be lost due to arriving in several consecutive bunch crossings. In addition, ATLAS and CMS perform trigger-based analyses relying either on triggering on accompanying "objects", e.g. missing transverse momentum, or by developing and deploying specialised triggers. The offline selection may require imposing additional cuts to reject cosmic-ray muons and/or enhance the reconstruction of highly-ionising particles.

Therefore, the efficiency may be lower and the probed parameter space may be reduced in ATLAS and CMS. MoEDAL, on the other hand, is mainly limited by the lower luminosity delivered at Point 8 , by the geometrical acceptance of the detectors, especially the MMTs, and by the requirement of passing the $Z / \beta$ threshold of NTDs. In general, ATLAS and CMS have demonstrated to cover high-velocities $\beta \gtrsim 0.2$, while MoEDAL is sensitive to ones with $\beta \lesssim 0.2$. Hence, by dropping selection criteria imposed by ATLAS and CMS when searching for long-lived SUSY particles, MoEDAL may extend the SUSY parameter space explored by LHC [49].

When discussing the detection of particles stopped (trapped) in material that they may decay later, different possibilities are explored. CMS and ATLAS look in empty bunch crossings for decays of trapped particles into jets. MoEDAL MMTs may be monitored in a underground/basement laboratory for tracks arising from such decays. The background in the latter case, coming from cosmic rays, should be easier to control and assess. The probed lifetimes should be larger due to the unlimited monitoring time.

\section{Summary and outlook}

MoEDAL is going to extend considerably the LHC reach in the search for (meta)stable highly ionising particles. The latter are predicted in a variety of theoretical models and include: magnetic monopoles, SUSY long-lived spartners, D-matter [50], quirks, strangelets, Q-balls, etc $[6,51]$. Such particles may be light enough to be producible at the LHC energies. In this paper we have described searches for monopoles and have discussed the MoEDAL relevance for long-lived partners in some SUSY models.

MoEDAL is optimised to probe precisely all such long lived states, unlike the other LHC experiments [4]. Furthermore it combines different detector technologies: plastic nuclear track detectors (NTDs), trapping volumes and pixel sensors [2]. The first physics results, pertaining to magnetic monopole trapping detectors, obtained with LHC Run 1 data [32], and the corresponding analysis at $13 \mathrm{TeV}$ have already been published [33]. The MoEDAL 
Collaboration is preparing new analyses with higher statistical precision from LHC Run 2, with other detectors (NTDs) and with a large variety of interpretations involving not only magnetic but also electric charges.

\section{Acknowledgments}

The author is grateful to the EXA2017 organisers for the kind invitation to present this talk. She acknowledges support by the Spanish MINEICO under the project FPA201565652-C4-1-R, by the Generalitat Valenciana through the MoEDAL-supporting agreement CON.21.2017-09.02.03 and the Excellence Project PROMETEO/2017/033, by the Severo Ochoa Excellence Centre Project SEV-2014-0398, and by a 2017 Leonardo Grant for Researchers and Cultural Creators, BBVA Foundation.

\section{References}

[1] The MoEDAL experiment web page: http://moedal.web.cern.ch/

[2] J. Pinfold et al. [MoEDAL Collaboration], "Technical Design Report of the MoEDAL Experiment," CERN-LHCC-2009-006, MoEDAL-TDR-001, and references therein.

[3] L. Evans and P. Bryant, JINST 3, S08001 (2008).

[4] A. De Roeck, A. Katre, P. Mermod, D. Milstead and T. Sloan, Eur. Phys. J. C 72, 1985 (2012).

[5] M. Fairbairn, A. C. Kraan, D. A. Milstead, T. Sjostrand, P. Z. Skands and T. Sloan, Phys. Rept. 438, 1 (2007); S. Burdin, M. Fairbairn, P. Mermod, D. Milstead, J. Pinfold, T. Sloan and W. Taylor, Phys. Rept. 582, 1 (2015).

[6] B. Acharya et al. [MoEDAL Collaboration], Int. J. Mod. Phys. A 29, 1430050 (2014).

[7] P. A. M. Dirac, Proc. Roy. Soc. Lond. A 133, 60 (1931).

[8] P. A. M. Dirac, Phys. Rev. 74, 817 (1948).

[9] G. 't Hooft, Nucl. Phys. B 79, 276 (1974); A. M. Polyakov, JETP Lett. 20, 194 (1974) [Pisma Zh. Eksp. Teor. Fiz. 20, 430 (1974)].

[10] B. Julia and A. Zee, Phys. Rev. D 11, 2227 (1975); Y. Nambu, Nucl. Phys. B 130, 505 (1977); E. Witten, Phys. Lett. 86B, 283 (1979); G. Lazarides, M. Magg and Q. Shafi, Phys. Lett. 97B, 87 (1980); R. d. Sorkin, Phys. Rev. Lett. 51, 87 (1983); D. J. Gross and M. J. Perry, Nucl. Phys. B 226, 29 (1983); J. S. Schwinger, Science 165, 757 (1969); J. Preskill, Ann. Rev. Nucl. Part. Sci. 34, 461 (1984); A. Achucarro and T. Vachaspati, Phys. Rept. 327, 347 (2000) [Phys. Rept. 327, 427 (2000)]; T. W. Kephart, C. A. Lee and Q. Shafi, JHEP 0701, 088 (2007); D. G. Pak, P. M. Zhang and L. P. Zou, Int. J. Mod. Phys. A 30, no. 27, 1550164 (2015); A. Rajantie, JHEP 0601, 088 (2006).

[11] V. Vento and V. S. Mantovani, "On the magnetic monopole mass," arXiv:1306.4213 [hep-ph] (2013).

[12] Y. M. Cho and D. Maison, Phys. Lett. B 391, 360 (1997); W. S. Bae and Y. M. Cho, J. Korean Phys. Soc. 46, 791 (2005).

[13] Y. M. Cho, K. Kimm and J. H. Yoon, Mod. Phys. Lett. A 31, no. 09, 1650053 (2016); Phys. Lett. B 761, 203 (2016).

[14] J. Ellis, N. E. Mavromatos and T. You, Phys. Lett. B 756, 29 (2016); J. Ellis, V. Sanz and T. You, JHEP 1407, 036 (2014).

[15] M. Barriola and A. Vilenkin, Phys. Rev. Lett. 63, 341 (1989).

[16] A. K. Drukier and S. Nussinov, Phys. Rev. Lett. 49, 102 (1982).

[17] P. O. Mazur and J. Papavassiliou, Phys. Rev. D 44, 1317 (1991); N. E. Mavromatos and J. Papavassiliou, Eur. Phys. J. C 78, no. 1, 68 (2018). 
[18] N. E. Mavromatos and S. Sarkar, Phys. Rev. D 95, no. 10, 104025 (2017).

[19] Y. B. Zeldovich and M. Y. Khlopov, Phys. Lett. 79B, 239 (1978).

[20] C. T. Hill, Nucl. Phys. B 224, 469 (1983).

[21] V. K. Dubrovich, Grav. Cosmol. Suppl. 8N1, 122 (2002).

[22] A. Rajantie, Contemp. Phys. 53, 195 (2012).

[23] A. Rajantie, Phys. Today 69, no. 10, 40 (2016).

[24] L. N. Epele, H. Fanchiotti, C. A. Garcia Canal and V. Vento, Eur. Phys. J. C 56, 87 (2008); Eur. Phys. J. C 62, 587 (2009).

[25] L. N. Epele, H. Fanchiotti, C. A. G. Canal, V. A. Mitsou and V. Vento, Eur. Phys. J. Plus 127, 60 (2012).

[26] L. N. Epele, H. Fanchiotti, C. A. G. Canal, V. A. Mitsou and V. Vento, "Can the $750 \mathrm{GeV}$ enhancement be a signal of light magnetic monopoles?," arXiv:1607.05592 [hep-ph] (2016).

[27] M. Kalliokoski, J. W. Lämsä, M. Mieskolainen and R. Orava, “Turning the LHC Ring into a New Physics Search Machine,” arXiv:1604.05778 [hep-ex] (2016).

[28] A. A. Alves, Jr. et al. [LHCb Collaboration], JINST 3, S08005 (2008).

[29] M. D. Joergensen, A. De Roeck, H.-P. Hachler, A. Hirt, A. Katre, P. Mermod, D. Milstead and T. Sloan, "Searching for magnetic monopoles trapped in accelerator material at the Large Hadron Collider," arXiv:1206.6793 [physics.ins-det] (2012).

[30] A. De Roeck, H. P. Hächler, A. M. Hirt, M.-D. Joergensen, A. Katre, P. Mermod, D. Milstead and T. Sloan, Eur. Phys. J. C 72, 2212 (2012).

[31] A. Sopczak et al., IEEE Trans. Nucl. Sci. 62, 3225 (2015).

[32] B. Acharya et al. [MoEDAL Collaboration], JHEP 1608, 067 (2016).

[33] B. Acharya et al. [MoEDAL Collaboration], Phys. Rev. Lett. 118, 061801 (2017).

[34] O. Gould and A. Rajantie, arXiv:1705.07052 [hep-ph] (2017).

[35] G. Aad et al. [ATLAS Collaboration], Phys. Rev. Lett. 109, 261803 (2012).

[36] G. Aad et al. [ATLAS Collaboration], Phys. Rev. D 93, 052009 (2016).

[37] L. Patrizii and M. Spurio, Ann. Rev. Nucl. Part. Sci. 65, 279 (2015).

[38] H. Goldberg, Phys. Rev. Lett. 50, 1419 (1983) Erratum: [Phys. Rev. Lett. 103, 099905 (2009)]; J. R. Ellis, J. S. Hagelin, D. V. Nanopoulos, K. A. Olive and M. Srednicki, Nucl. Phys. B 238, 453 (1984).

[39] V. A. Mitsou, PoS PLANCK 2015, 085 (2015).

[40] J. R. Ellis, K. A. Olive, Y. Santoso and V. C. Spanos, Phys. Lett. B 565, 176 (2003).

[41] O. Buchmueller et al., Eur. Phys. J. C 72, 2243 (2012); Eur. Phys. J. C 74, 2922 (2014).

[42] T. Jittoh, J. Sato, T. Shimomura and M. Yamanaka, Phys. Rev. D 73, 055009 (2006) Erratum: [Phys. Rev. D 87, 019901 (2013)].

[43] S. Kaneko, J. Sato, T. Shimomura, O. Vives and M. Yamanaka, Phys. Rev. D 78, 116013 (2008) Erratum: [Phys. Rev. D 87, 039904 (2013)].

[44] J. R. Ellis, K. A. Olive and Y. Santoso, JHEP 0810, 005 (2008).

[45] J. L. Diaz-Cruz, J. R. Ellis, K. A. Olive and Y. Santoso, JHEP 0705, 003 (2007).

[46] K. Hamaguchi, M. M. Nojiri and A. de Roeck, JHEP 0703, 046 (2007).

[47] J. L. Feng, S. Iwamoto, Y. Shadmi and S. Tarem, JHEP 1512, 166 (2015).

[48] N. Arkani-Hamed and S. Dimopoulos, JHEP 0506, 073 (2005); G. F. Giudice and A. Romanino, Nucl. Phys. B 699, 65 (2004) Erratum: [Nucl. Phys. B 706, 487 (2005)].

[49] J. Mamuzic, N. E .Mavromatos, V. A. Mitsou, J. L. Pinfold, R. Ruiz de Austri, K. Sakurai, O. Vives, "Long-lived charged supersymmetric particles and MoEDAL," in preparation. 
[50] G. Shiu and L. T. Wang, Phys. Rev. D 69, 126007 (2004); J. R. Ellis, N. E. Mavromatos and D. V. Nanopoulos, Gen. Rel. Grav. 32, 943 (2000); Phys. Lett. B 665, 412 (2008); J. R. Ellis, N. E. Mavromatos and M. Westmuckett, Phys. Rev. D 70, 044036 (2004); ibid. 71, 106006 (2005); N. E. Mavromatos, S. Sarkar and A. Vergou, Phys. Lett. B 696, 300 (2011); N. E. Mavromatos, V. A. Mitsou, S. Sarkar and A. Vergou, Eur. Phys. J. C 72, 1956 (2012).

[51] V. A. Mitsou [MoEDAL Collaboration], EPJ Web Conf. 95, 04042 (2015); PoS EPS-HEP2015, 109 (2015); PoS CORFU 2016, 028 (2017); N. E. Mavromatos and V. A. Mitsou [MoEDAL Collaboration], EPJ Web Conf. 164, 04001 (2017). 